\title{
On 3-Stage Clos Networks with Different Nonblocking Requirements on Two Types of Calls
}

\author{
HUNG-LIN FU* \\ Department of Applied Mathematics, National Chiao Tung University, Hsin Chu, Taiwan, R.O.C. \\ FRANK K. HWANG ${ }^{\dagger}$ \\ fhwang@math.nctu.edu.tw \\ Department of Applied Mathematics, National Chiao Tung University, Hsinchu 300, Taiwan, R.O.C.
}

Received February 21, 2005; Accepted February 21, 2005

\begin{abstract}
Hwang and Lin introduced a new nonblocking requirement for 2-cast traffic which imposes different requirements on different types of coexisting calls. The requirement is strictly nonblocking for point-to-point calls among the 2-cast traffic, and is rearrangeable for genuine 2-cast calls. They conjectured that the 3-stage Clos network $C(n, n, r, r, 2 n)$ satisfies the above requirement. We prove that $C(n, n, 4, r, 2 n)$ satisfies the above requirement.
\end{abstract}

Keywords: 3-stage Clos networks, nonblocking networks, rearrangeable networks

\section{Introduction}

Consider a 3-stage Clos network $C\left(n_{1}, n_{2}, r_{1}, r_{2}, m\right)$ where the input-stage consists of $r_{1}$ $n_{1} \times m$ crossbars, the middle-stage $m r_{1} \times r_{2}$ crossbars, the output-stage $r_{2} m \times n_{2}$ crossbars and there exists one link between every pair of switches between two adjacent stages (see figure 1).

The inlets of the input switches are the inputs of the network and the outlets of the output switches are the outputs of the network. A network is called strictly nonblocking if any pair of idle input and output can be connected regardless of the existing connections of other pairs in the network (all paths must be link disjoint). A network is called rearrangeable if any set of disjoint pairs of inputs and outputs can be simultaneously connected. If the calls come sequentially, rearrangeability means we can disconnect existing connections and reroute them together with the new call simultaneously.

Besides the point-to-point call as mentioned above, there is also the multicast call where an input can request connection to many outputs. A multicast call is called $f$-cast if at most $f$ outputs can be requested in a connection. Although technically a point-to-point call is also a multicast call, in this paper the term $f$-cast call refers to a call with more than one output.

*Supported in part by NSC91-2115-M009-002.

${ }^{\dagger}$ Supported in part by the National Science Council under grant NSC91-2115-M009-010 and by the Li-Li-Tai-Yang Network Research Center of National Chiao Tung University. 


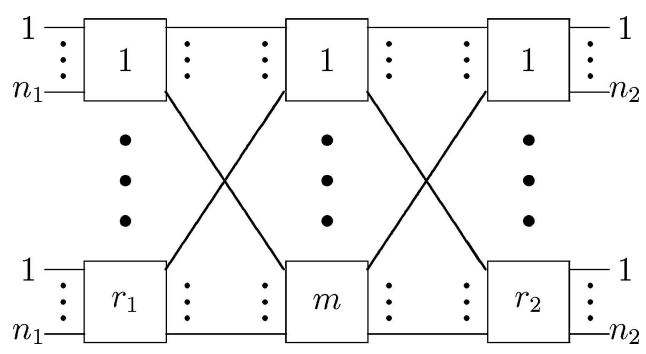

Figure 1. $C\left(n_{1}, n_{2}, r_{1}, r_{2}, m\right)$.

Hwang and Lin (1995) studied a real problem where the topology of the network is the 3-stage Clos network and the main feature is point-to-point strictly nonblocking. However, the builder of this network wishes to claim a secondary feature that the network can take care of some multicast calls which come up occasionally. More specifically, the secondary feature is that all 2-cast calls can be routed in a rearrangeably nonblocking fashion, i.e., a set $S$ of existing connections may need to be taken down, and then reconnected, possibly through different paths, along with the current 2-cast request. We will call this mixture of requirement the $H L$-requirement. They conjectured:

Conjecture. $C(n, n, r, r, 2 n)$ satisfies the $H L$-requirement.

Although it is well known (Masson and Jordan, 1972) that $C(n, n, r, r, 2 n)$ is rearrangeable if all calls are 2-cast, and is also strictly nonblocking (Clos, 1953) if all calls are point-to-point, the difficulty in satisfying the $H L$-requirement is that the routing guaranteeing the "rearrangeable" part employs input switch fan-out capability, while the routing guaranteeing the "strictly nonblocking" part forbids input switch fan-out. Thus to preserve the strict nonblockingness property, we must find a new rearrangeable routing for the 2-cast calls not using the input switch fan-out. In other words, we might as well assume that the input switches have no fan-out capability (we use $C^{\prime}\left(n_{1}, n_{2}, r_{1}, r_{2}, m\right)$ to denote such a Clos network).

Recently, Du and Ngo (2002) proved the conjecture for $n_{1} \geq n_{2}=2$ or 3 . Hwang et al. (2002) proved it for $n_{1}>n_{2}$ and $r_{2} \leq 4$. Note that for $r_{2}=4$, there are at most $2 n$ 2-cast calls due to the restriction on the number of outputs, and this fact was crucially used in their proof. In this paper, we will prove the case where $r_{1}=4$ but $r_{2}$ is unrestricted. Therefore, there can be as many as $4 n 2$-cast calls in the network.

\section{An equivalent graph-coloring problems}

Since $C^{\prime}\left(n_{1}, n_{2}, r_{1}, r_{2}, m\right)$ has the property that each request, be it point to point or multicast, consumes only one outlink of its input switch, the necessary and sufficient condition for point-to-point strictly nonblocking remains the same as $C\left(n_{1}, n_{2}, r_{1}, r_{2}, m\right)$ (Clos, 1953).

Lemma 1. $C^{\prime}\left(n_{1}, n_{2}, r_{1}, r_{2}, m\right)$ is strictly nonblocking for all point-to-point calls in a multicast traffic if $m \geq \min \left\{n_{1}+n_{2}-1, n_{1} r_{1}, n_{2} r_{2}\right\}$. 
Therefore, for $m$ satisfying the condition in Lemma 1, we need only be concerned with the rearrangeability of 2-cast calls. Since a 2-cast call where the two outputs are on the same output switch can be connected by a single path going to that output switch and then splitting to the two outputs using the output switch fan-out, the worst case is when each 2-cast call involves two distinct output switches.

We first transform the network problem into a graph-coloring problem. Let $G$ denote the graph whose vertices are the output switches and whose edges are the output-pairs of the 2-cast calls. $G$ can also be viewed as the union of four edge-disjoint graphs where $G_{i}$ consists of the set of edges corresponding to 2-cast calls from input switch $i$. Then the problem is to color the edges in $2 n$ colors such that edges from the same vertex must be in different colors, and edges from the same $G_{i}$ must also be in different colors.

Note that each color represents a distinct middle switch, and an edge $e$ in color $c$ means the 2-cast call $e$ will be routed through the middle switch $c$. Since all edges in the same color are from different $G_{i}$ (input switches) and different vertices (output switches), they can go through the same middle switch without conflict.

\section{The main results}

Consider another graph $G^{\prime}$ whose nodes are the edges of $G$, and a link exists between node $\{x, y\}$ and node $\{u, v\}$ if and only if $\{x, y\} \cap\{u, v\}=\phi$. Decompose $G^{\prime}$ into two node-disjoint subgraphs $G_{o}^{\prime}$ and $G_{e}^{\prime}$ where nodes of $G_{o}^{\prime}$ are edges in $G_{1} \cup G_{3}$, and nodes of $G_{e}^{\prime}$ are edges in $G_{2} \cup G_{4}$. We prove

Lemma 2. Suppose $G$ contains $4 n$ edges. Then there exists a perfect matching between the $2 n$ nodes in $G_{o}^{\prime}$ and the $2 n$ nodes of $G_{e}^{\prime}$.

Proof: Consider a set $K$ of $k$ nodes in $G_{o}^{\prime}$. Let $S$ be the set of nodes in $G_{e}^{\prime}$ which are connected to $K$. We prove $|S| \geq k$. Lemma 2 then follows from the well known Hall's theorem on system of distinct representatives (Hall, 1935).

Suppose to the contrary $|S|<k$. Let $\bar{S}$ denote the complementary set of $S$ in $G_{e}^{\prime}$. Then $|\bar{S}|+|K| \geq 2 n+1$. Hence either $|\bar{S}|$ or $|K| \geq n+1$. Without loss of generality, assume $k \geq n+1$. Let node $\{x, y\} \in \bar{S}$. Then every node of $K$ must contain at least one element of $\{x, y\}$. This implies at least one node of $\bar{S}$ contains neither $x$ nor $y$ for otherwise the total appearances of $x$ and $y$ would be at least $2 n+1$ while there are only $n$ of each. Let $\{u, v\}$ be a node not intersecting $\{x, y\}$. Then every node of $K$ must contain either $u$ or $v$, which implies that every node of $K$ is of the form $\{x, y\} \times\{u, v\}$. Furthermore, since there are only $n-1 x$ (respectively, $y, u, v$ ) available for nodes in $K$, at least one node in $K$ does not contain $x$ (respectively, $y, u, v$ ). Hence each node in $\bar{S}$ must intersect $\{x, y, u, v\}$ in two symbols since if it intersects only in one symbol, say $x$, then it would not intersect the node in $K$ not containing $x$. Therefore every node in $K \cup \bar{S}$ contains two symbols of $\{x, y, u, v\}$. But this is impossible since $K \cup \bar{S}$ requires at least $2(2 n+1)$ symbols of $\{x, y, u, v\}$ while only $4 n$ are available.

Theorem 3. $C^{\prime}(n, n, 4, r, 2 n)$ satisfies the HL-requirement. 
Proof: For $r \geq 8, G$ has $4 n$ edges. Theorem 3 then follows from Lemmas 1 and 2. For $r<8$, we artificially add $8-r$ output switches and 2-cast calls between idle inputs and outputs such that the total number of 2-cast calls is $4 n$. By Lemma 2, there exists a routing of the 2-cast calls for the enhanced network. Delete all connections involving the added calls or the added output switches to obtain a routing for the original network.

\section{Conclusions}

The problem studied in this paper is interesting in three aspects. On the network theory aspect, Kirkpatrick et al. (1955) has studied the broadcast rearrangeable $C^{\prime}\left(n_{1}, n_{2}, r_{1}, r_{2}, m\right)$, i.e., a 3-stage Clos network whose input switches have no fan-out capability. We continued this line of study for $f$-cast traffic. On the network application aspect, it deals with a new model in which nonblocking property is required on the predominant type of calls, but a weaker property is required on the accidental type of calls. The need of this separate treatment is practical and reasonable. The third aspect is its relation with graph coloring. Since the $H L$-requirment can be translated to a graph-theoretic problem as exposed in Section 2, it has stimulated research into graph-coloring problems where the constraints come not just from the usual "adjacency", but also a second source ( $G_{i}$ in our problem). See (Du et al., 1993; Du and Ngo, 2002; Fleischner and Stiebitz, 1992) for more detailed discussion on that.

\section{References}

C. Clos, “A study of nonblocking switching networks," Bell Syst. Tech. J., vol. 32, pp. 406-424, 1953.

D.Z. Du, D.F. Hsu, and F.K. Hwang, "The hamiltonian property of consecutive-d digraphs," Mathl. Comput. Modelling, vol. 17, pp. 61-63, 1993.

D.Z. Du and H.Q. Ngo, "An extension of DHH-Erdös conjecture on cycle-plus-triangle graphs," Taiwan J. Math. vol. 6, pp. 65-105, 2002.

H. Fleischner and M. Stiebitz, "A solution to a coloring problem of P. Erdös,” Disc. Math., vol. 101, pp. 39-48, 1992.

P. Hall, "On representatives of subsets," J. London Math. Soc., vol. 10, pp. 26-30, 1935.

F.K. Hwang, S.-C. Liaw, and L.D. Tong, "Strictly nonbolcking 3-stage Clos networks with some rearrangeable multicast capability," IEEE Trans. Commun., vol. 6, pp. 261-267, 2002.

F.K. Hwang and C.H. Lin, "Broadcasting in a three-stage point-to-point nonblocking network," Inter. J. Rel. Qual. Safety Eng., vol. 2, pp. 299-307, 1995.

D.G. Kirkpatrick, M. Klawe, and N. Pippenger, "Some graph-coloring theorems with applications to generalized connection networks," SIAM J. Alg. Disc. Methods, vol. 6, pp. 576-582, 1955.

G.M. Masson and B.W. Jordan, "Generalized multi-stage connection networks," Networks, vol. 2, pp. 191-209, 1972. 